\section{Making the most out of the minimum: atom probe tomography's role in characterising returned extraterrestrial samples.}

\author{
LUKE DALY $Y^{1,2,3,4}$, MARTIN LEE ${ }^{1}$, LYDIA HALLIS ${ }^{1}$, \\ JOSHUA FRANZ EINSLE ${ }^{1}$, PHILLIP A BLAND ${ }^{3}$, DAVID W \\ SAXEY $^{3}$, STEVEN M REDDY $^{3}$, DENIS FOUGEROUSE ${ }^{3}$, \\ WILLIAM D A RICKARD ${ }^{3}$, JULIE CAIRNEY ${ }^{2}$, INGRID \\ MCCARROLL $^{2}$, LIMEI YANG ${ }^{2}$, PAUL A J BAGOT ${ }^{4}$ AND \\ JAMES R DARLING ${ }^{5}$ \\ ${ }^{1}$ University of Glasgow \\ ${ }^{2}$ University of Sydney \\ ${ }^{3}$ Curtin University \\ ${ }^{4}$ University of Oxford \\ ${ }^{5}$ University of Portsmouth \\ Presenting Author: luke.daly@glasgow.ac.uk
}

Atom probe tomography (APT) is a relatively new technique in planetary science [1] that enables the collection of atomicscale chemical, isotopic and spatial information in 3D from small sample volumes $\left(<0.01 \mu \mathrm{m}^{3}\right.$ [2-3]). As such, APT has the potential to provide unique insights into the nanoscale heterogeneity of extraterrestrial materials. [1,2,4-6].

In this talk we will provide a brief overview of the APT technique, and sample preparation protocols [4] including the effectiveness of using a cryo-focused ion beam transfer mechanism to minimize volatile element loss from hydrous samples. We give examples of recent key advances in developing APT for its application to extraterrestrial materials that have been, and will be delivered to Earth from asteroids, the Moon, and Mars [1,2,4-11] by sample return missions. Specifically, we will outline the how APT has been used to directly detect water molecules in the space weathered surfaces of mineral grains from the asteroid Itokawa [12], water rock reaction interfaces in Martian nakhlite meteorites, and the first APT measurement of phyllosilicates in a terrestrial peridotite [13] and CM chondrite meteorites (Figure 1). Our work demonstrates that APT measurements, particularly when coordinated with conventional microanalytical techniques, are providing a new avenue to investigate extraterrestrial processes from asteroid impacts to space weathering [1,2,4-11].

References: [1] Heck et al., (2014) MAPS, 49, 453-467. [2] Daly et al., (2020) IOP Conf. Ser. Mat. Sci. \& Eng., 891, 012008. [3] Reddy et al., $2020 G \& G R, 44(1), 5-50$. [4] Rickard et al., (2020) $M \& M, 26(4), 750-757$, [5] White et al., (2020) PNAS, 117(21), 11217-11219. [6] Greer et al., (2020) MAPS, 55(2), 426-440. [7] Lewis et al., (2015) Ultramic. 159, 248-254. [8] Einsle et al., (2018) PNAS, 115(49), E11436-E11445. [9] White et al., (2017) Nat. Comms. [10] Darling et al., (2021) GCA, 293, 422-437. [11] Lewis et al., (2020) MAPS, 55(6), 1382-1403. [12] Daly et al., (in review) Nat. Astro. [13] Daly et al., (in review) $G \& G R$.

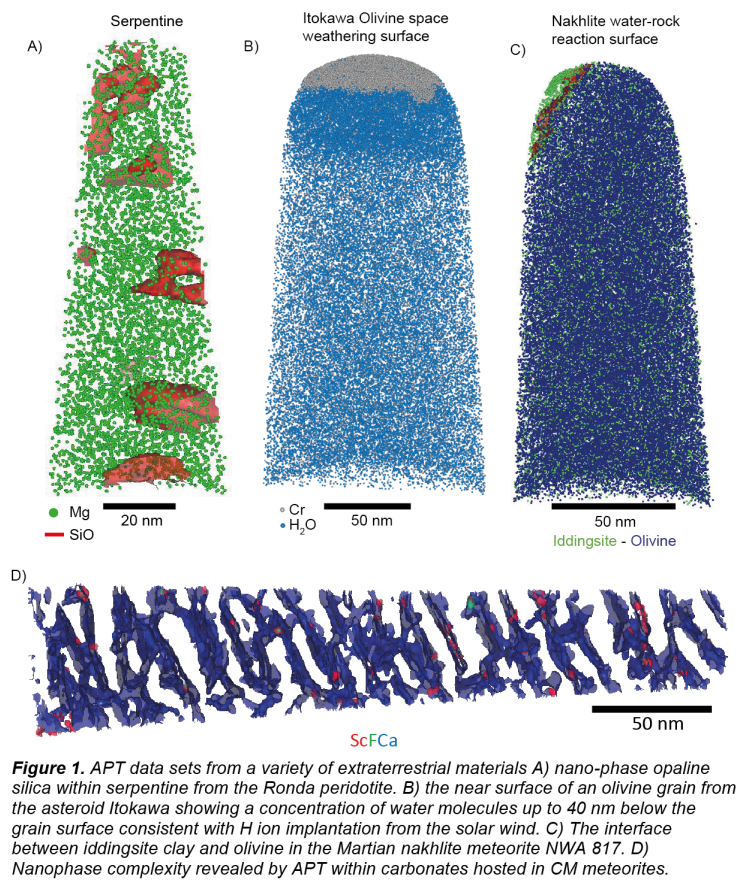

between iddingsite clay and olivine in the Martian nakhlite meteorite NWA 817. D) 